\title{
Multivariate risks and depth-trimmed regions
}

\author{
Ignacio Cascos • Ilya Molchanov
}

Received: 21 June 2006 / Accepted: 12 March 2007 /

Published online: 15 May 2007

(C) Springer-Verlag 2007

\begin{abstract}
We describe a general framework for measuring risks, where the risk measure takes values in an abstract cone. It is shown that this approach naturally includes the classical risk measures and set-valued risk measures and yields a natural definition of vector-valued risk measures. Several main constructions of risk measures are described in this axiomatic framework.

It is shown that the concept of depth-trimmed (or central) regions from multivariate statistics is closely related to the definition of risk measures. In particular, the halfspace trimming corresponds to the Value-at-Risk, while the zonoid trimming yields the expected shortfall. In the abstract framework, it is shown how to establish a both-ways correspondence between risk measures and depth-trimmed regions. It is also demonstrated how the lattice structure of the space of risk values influences this relationship.
\end{abstract}

Keywords Acceptance set $\cdot$ Cone $\cdot$ Depth-trimmed region $\cdot$ Multivariate risk $\cdot$ Risk measure

Mathematics Subject Classification (2000) 91B30 · 91B82 - 60D05 - 62H99

JEL Classification C60 - C61

I. Cascos supported by the Spanish Ministry of Education and Science Grant MTM2005-02254.

I. Molchanov supported by Swiss National Science Foundation Grant 200020-109217.

\section{Cascos}

Department of Statistics, Universidad Carlos III de Madrid, Av. Universidad 30,

E-28911 Leganés, Madrid, Spain

e-mail: ignacio.cascos@uc3m.es

I. Molchanov ( $\varangle)$

Department of Mathematical Statistics and Actuarial Science, University of Berne,

Sidlerstr. 5, CH-3012 Berne, Switzerland

e-mail: ilya.molchanov@stat.unibe.ch 


\section{Introduction}

Risk measures are widely used in financial engineering to assess the risk of investments and to optimise the capital allocation. The modern theory of coherent risk measures [2,9] aims to derive properties of risk measures from several basic axioms: translation-invariance, monotonicity, homogeneity, and convexity. The risk measures are mostly considered in the univariate case, i.e., it is assumed that all assets have been transferred to their monetary values. The quantile-based risk measures gain a particular importance in the form of so-called spectral risk measures that are weighted integrals of the quantile function, see [1].

When assessing risks of multivariate portfolios, the situation becomes more complicated. The quantile function is not a numerical function any more, and it is not possible to represent all portfolios as functions of a uniform random variable. The simplest approach to assess the risk of a multivariate portfolio is to aggregate the individual assets using their cash equivalents and then assess the risk of the combined univariate portfolio. Then all portfolios with identically distributed monetary equivalents would have identical risks.

Several recent papers suggest various alternative ways of measuring risks for multivariate portfolios without taking their monetary equivalents. The multivariate analogue of the Value-at-Risk discussed in [10] is based on set-valued quantiles of the multivariate cumulative distribution function. A construction of real-valued multivariate risk measures based on combining univariate risks from transformed portfolios is described in [4]. Multivariate coherent risk measures have been studied in [16] following the techniques from [9] based on the duality representations. The risk measures considered in [16] are actually set-valued, and the preference order corresponds to the ordering of sets by inclusion. It is interesting to note that this order has the same meaning for risk but formally is the exact opposite to the ordering of univariate risks from [2]. Set-valued risk measures have been also studied in [12].

Because of this reason and in order to unify several existing definitions, we decided to consider risk measures as maps that take values in a certain partially ordered cone, which may be, e.g., the real line or the Euclidean space or the family of convex sets in the Euclidean space. We single out the basic properties of so defined risk measures and then describe the main technical constructions that make it possible to produce new risk measures from the existing ones while respecting their properties, e.g., the homogeneity or coherence. It is not always assumed that the risk measures are coherent. Note that risk measures with values in a partially ordered cone have been considered in [15], where however it was assumed that this cone is embeddable into a linear space. This is not the case for set-valued risk measures which are also covered by the current work. These set-valued measures can be used to produce vector-valued or real-valued risk measures for multivariate portfolios.

In comparison with the studies of multivariate risk measures, multivariate statistical theory has an impressive toolbox suitable to handle random vectors. We show that the multivariate setting for the risk measures has a number of common features with the concept of central (or depth-trimmed) regions well known in multivariate statistics $[24,25]$. They associate a random vector with a set formed by the points in space located near the "central value" of this random vector. The risk measure is generated by considering all translations of a random vector that bring its central region to the 
positive (acceptable) part of the space. In other words, the riskiness is determined by the relative location of the central region compared to the acceptable or completely nonacceptable risks. Note that in the multivariate setting the sets of acceptable and nonacceptable risk values are no longer complementary, as they are in the univariate setting. Estimation methods for depth-trimmed regions may then be utilised to come up with estimators for multivariate risk measures. Despite the fact that the definition of central regions (and indeed the name also) treats all directions in the same way, it is possible to establish a two-way link between depth-trimmed regions and risk measures.

The paper is organized as follows. Section 2 introduces the concept of a risk measure with values in an abstract cone. As special cases, one obtains the classical risk measures [2], set-valued risk measures of [16], and vector-valued risk measures. A crucial concept here is the function that assigns risks to deterministic outcomes and controls changes of the risk if a deterministic amount is being added to a portfolio. The partial order relation on the space of risks makes it possible to consider it as a lattice.

The acceptance cone constitutes a subset of acceptable values for the risk measure, while the acceptance set is the family of random vectors whose risks belong to the acceptance cone. Section 3 discusses the main properties of the acceptance set and the acceptance cone. We single out conditions that make it possible to retrieve the risk measure from the acceptance set it generates. This self-consistency condition can be traced to some facts from the morphological theory of lattices [13].

Section 4 describes several ways to construct new risk measures: minimization, re-centering, homogenization, worst conditioning, and transformations of risks. In particular, the worst conditioning is a generic construction that yields the expected shortfall if applied to the expectation. It is shown that by transforming risks it is possible to produce vector-valued risk measures from set-valued risk measures. This construction can be applied, for instance, to the set-valued risk measures from [16].

The definition of depth-trimmed regions and their essential properties in view of relationships to risk measures are given in Sect. 5. In particular, the well-known halfspace trimmed regions [20, 23] correspond to the Value-at-Risk, and the zonoid trimming $[18,22]$ produces the expected shortfall. This analogy goes much further and leads to a systematic construction of a risk measure from a family of depth-trimmed regions in Sect. 6. The main idea here is to map the depth-trimmed region of a random vector into the risk space using the function that assigns risks to deterministic outcomes and then consider all translations of the image (of the depth-trimmed region) that place it inside the acceptance cone. Examples of basic risk measures obtained in this way are described in Sect. 7. It is shown in Sect. 8 that the correspondence between risk measures and depth-trimmed regions goes both ways, i.e., it is possible to construct a family of depth-trimmed regions from a risk measure, so that, under some conditions, the initial risk measure is recoverable from the obtained family of depth-trimmed regions.

Finally, Sect. 9 deals with dual representations of coherent risk measures and depth-trimmed regions using families of measures, in a way similar to the well-known approach [9] for real-valued coherent risk measures. In particular we show that all coherent vector-valued risk measures for the coordinatewise order are marginalised, i.e., can be represented as the vector of risk measures for the marginals. This fact confirms 
the idea that set-valued risk measures are a natural tool for multivariate portfolios if one is interested in nontrivial coherent risk measures.

\section{Risk measures in abstract cones}

A risky portfolio is modeled as an essentially bounded random vector $X$ that represents a financial gain. Let $L_{d}^{\infty}$ denote the set of all essentially bounded $d$-dimensional random vectors on a probability space $(\Omega, \mathfrak{F}, \mathbf{P})$. In order to combine several definitions of risk measures, it is sensible to regard them as functionals on $L_{d}^{\infty}$ with values in a partially ordered convex cone $\mathbb{G}$.

Definition 2.1 (Semigroup and convex cone) An Abelian topological semigroup is a topological space $\mathbb{G}$ equipped with a commutative and associative continuous binary operation $\oplus$. It is assumed that $\mathbb{G}$ possesses a neutral element e satisfying $x \oplus \mathbf{e}=x$ for all $x \in \mathbb{G}$. The semigroup $\mathbb{G}$ is a convex cone if it is also equipped with a continuous operation $(x, t) \mapsto t \odot x$ of multiplication by positive scalars $t>0$ for $x \in \mathbb{G}$ so that $1 \odot x=x$ for all $x \in \mathbb{G}, t \odot \mathbf{e}=\mathbf{e}$ for all $t>0$, and the following conditions are satisfied:

$$
\begin{aligned}
& t \odot(x \oplus y)=t \odot x \oplus t \odot y, \quad t>0, x, y \in \mathbb{G}, \\
& t \odot(s \odot x)=(t s) \odot x, \quad t, s>0, x \in \mathbb{G} .
\end{aligned}
$$

Assume throughout that $\mathbb{G}$ is endowed with a partial order $\preceq$ that is compatible with the (commutative) addition operation and multiplication by scalars, i.e., $x \preceq y$ implies that $x \oplus z \preceq y \oplus z$ for all $z$ and $t \odot x \preceq t \odot y$ for all $t>0$. Furthermore, assume that $\mathbb{G}$ with the order $\preceq$ is a complete lattice, i.e., every set has supremum and infimum, which are denoted by $\vee$ and $\wedge$, respectively. Since this partial order may differ from the conventional order for real numbers, we retain the notation supremum and infimum (also min and max) for the conventional order on the real line, while $\checkmark$ and $\wedge$ denote the supremum and infimum in $\mathbb{G}$. The top element of $\mathbb{G}$ is denoted by $\mathbb{T}$. It is assumed that the top element is absorbing, i.e., $\mathbb{T} \oplus a=\mathbb{T}$ for all $a \in \mathbb{G}$.

Note that the cone $\mathbb{G}$ is not necessarily embeddable in a linear space, since the addition operation does not necessarily obey the cancellation law and the second distributivity law $t \odot x \oplus s \odot x=(t+s) \odot x$ is not imposed; see [8] for a discussion of algebraic properties of convex cones. Accordingly, it is not possible to view $\mathbb{G}$ as a partially ordered linear space. This situation is typical if $\mathbb{G}$ is the family of convex sets in the Euclidean space $\mathbb{R}^{d}$ and the additive operation is the closed Minkowski addition, i.e., the sum $A \oplus B$ of two sets is the topological closure of $\{x+y: x \in A, y \in B\}$. Note that the Minkowski sum of two noncompact closed sets is not necessarily closed. The multiplication by positive numbers is given by $t A=\{t x: x \in A\}$, i.e., the usual dilation of $A$ by $t>0$, and we simply write $x+A$ instead of $\{x\} \oplus A$.

We retain the usual + and multiplication signs for operations with real numbers and vectors in $\mathbb{R}^{d}$. For convenience, letters $x, y, z$ with or without subscripts stand for points in $\mathbb{R}^{d}$, letters $t, s$ represent real numbers, letters $a, b$ denote elements of 
$\mathbb{G}$, letters $X, Y$ are used for random variables or random vectors, and $A, B, F, K$ are subsets of $\mathbb{R}^{d}$.

A proper Euclidean convex cone $K$ is a strict subset of $\mathbb{R}^{d}$ such that $\mathbb{R}_{+}^{d} \subseteq K$, $K$ does not contain any line, and $x+y \in K, t x \in K$ for all $x, y \in K$ and $t>0$. In the univariate case $(d=1)$, the only possibility is $K=[0, \infty)$.

Definition 2.2 (Order in $\mathbb{R}^{d}$ ) Let $K$ be a proper Euclidean convex cone. For $x, y \in \mathbb{R}^{d}$, we write $x \leq_{K} y$ if and only if $y-x \in K$.

From the economic viewpoint, this ordering would correspond, e.g., to exchanges of various currencies, cf. [16, 17].

A risk measure is a functional on $L_{d}^{\infty}$ with values in $\mathbb{G}$. As the first step of its proper definition, one should specify how this functional acts on degenerate random variables, i.e., on the space $\mathbb{R}^{d}$, which is naturally embedded in $L_{d}^{\infty}$. This action is defined by a function $f: \mathbb{R}^{d} \mapsto \mathbb{G}$, which is interpreted as the risk associated with the degenerate random variable $X=x$ a.s. Assume that $f(0)=\mathbf{e}, f$ is linear, i.e.,

$$
f(x) \oplus f(y)=f(x+y)
$$

for all $x, y \in \mathbb{R}^{d}$, and nondecreasing, i.e., $f(y) \preceq f(x)$ if $y \leq_{K} x$. The mapping $f$ is a linear positive map between the partially ordered linear spaces $\mathbb{R}^{d}$ with the $\leq_{K}$ order and the space $\mathbb{F}=\left\{f(y): y \in \mathbb{R}^{d}\right\}$ with the order inherited from $\mathbb{G}$. Condition (2.1) implies that $f(x) \neq \mathbb{T}$ for all $x$. Indeed, if $f(x)=\mathbb{T}$, then $f(x+y)=\mathbb{T}$ for all $y$, so that $f$ identically equals $\mathbb{T}$ contrary to the fact that $f(0)=\mathbf{e}$.

The following definition specifies the desirable properties of risk measures.

Definition 2.3 (Risk measure) A functional $\varrho: L_{d}^{\infty} \mapsto \mathbb{G}$ is called a risk measure associated with $f$ if $\varrho(X)=f(x)$ in case $X=x$ a.s. and the following conditions hold:

$\mathrm{R} 1 f(y) \oplus \varrho(X)=\varrho(X+y)$ for all $y \in \mathbb{R}^{d}$;

R2 $\varrho(Y) \preceq \varrho(X)$ whenever $Y \leq_{K} X$ a.s.

It is called a homogeneous risk measure if also

$\mathrm{R} 3 \varrho(t X)=t \odot \varrho(X)$ for all $t>0$ and $X \in L_{d}^{\infty}$

and a coherent risk measure if additionally

$\mathrm{R} 4 \varrho(X) \oplus \varrho(Y) \preceq \varrho(X+Y)$ for all $X, Y \in L_{d}^{\infty}$.

Condition R2 means that $\varrho$ is a lattice morphism between $L_{d}^{\infty}$ with the partial order generated by $\leq_{K}$ and $\mathbb{G}$. It is also possible to consider not necessarily homogeneous risk measures that satisfy the assumption

$$
t \odot \varrho(X) \oplus(1-t) \odot \varrho(Y) \preceq \varrho(t X+(1-t) Y)
$$

for all $t \in[0,1]$, which are traditionally called convex [11] (despite the fact that the inequality in our setting actually means that $\varrho$ is concave). 
Note that the multiplication by numbers in $\mathbb{G}$ is not needed if R3 is not considered. In this case, one can only require that $\mathbb{G}$ is a partially ordered Abelian semigroup. Furthermore, Definition 2.3 can be formulated for any partially ordered cone $\mathbb{G}$ (not necessarily a complete lattice) and any partial ordering on $\mathbb{R}^{d}$.

Since $\mathbf{e} \neq \mathbb{T}$, the condition $f(0)=\mathbf{e}$ together with R2 implies that $\varrho(X)$ never takes the value $\mathbb{T}$. This corresponds to the requirement that conventional risk measures do not take the value $-\infty$, see [9]. Indeed, if $\varrho(X)=\mathbb{T}$, then $f(a)=\varrho(a)=\mathbb{T}$ for $a$ being an upper bound for $X$.

The use of the function $f$ in Definition 2.3 is twofold. It determines risks of deterministic portfolios and also controls how the risk of $X$ changes if a deterministic quantity is added to the portfolio $X$. The second task can be also delegated to another function $g: \mathbb{R}^{d} \mapsto \mathbb{G}$, so that R1 becomes $g(y) \oplus \varrho(X)=\varrho(X+y)$ and $g(y) \oplus g(-y)=\mathbf{e}$ for all $y \in \mathbb{R}^{d}$. It is easy to show that $f$ and $g$ coincide if and only if $f(0)=\mathbf{e}$.

Example 2.4 (Set-valued risk measures) Consider the family of closed convex sets in $\mathbb{R}^{d}$ partially ordered by inclusion with the addition defined as the closed Minkowski sum and the conventional dilation by positive numbers. Define $f(x)=$ $\left\{y \in \mathbb{R}^{d}:-x \leq_{K} y\right\}=-x+K$, where $K$ is a proper Euclidean cone from Definition 2.2. In particular, the fact that $\varrho(X) \supset K$ means that $X$ has a negative risk. In this case, Definition 2.3 turns into [16, Definition 2.1]. Since $f(0)=K$ has to be the neutral element, the relevant cone $\mathbb{G}$ should consist of all closed convex sets $F \subseteq \mathbb{R}^{d}$ such that the closed Minkowski sum $F \oplus K$ coincides with $F$. This important family of sets will be denoted by $\mathbb{G}_{K}$.

Let us show that $\mathbb{G}_{K}$ is a complete lattice. Consider any family of sets $\left\{A_{i}: i \in I\right\} \subseteq \mathbb{G}_{K}$. Then $F=\bigvee_{i \in I} A_{i}$ is the smallest convex set that contains all the $A_{i}$, i.e., $F$ is the closure of the convex hull of the union of these sets. Since $F$ is closed convex and $F=\bigvee\left(A_{i} \oplus K\right)=K \oplus F$, we have $F \in \mathbb{G}_{K}$. Furthermore, $M=\bigwedge_{i \in I} A_{i}$ is given by $M=\cap_{i \in I} A_{i}$. The set $M$ is closed convex and also belongs to $\mathbb{G}_{K}$, since

$$
M=\bigcap_{i \in I} A_{i}=\bigcap_{i \in I}\left(A_{i} \oplus K\right) \supseteq K \oplus \bigcap_{i \in I} A_{i} \supseteq M,
$$

because $K$ contains the origin.

Example 2.5 (Univariate risk measures) The classical definition of real-valued coherent risk measures from Artzner et al. [2] can be recovered from the setting of Example 2.4 for $d=1$ and $\varrho(X)=[\rho(X), \infty)$, where $\rho(X)$ is the risk measure of $X$ as in [2]. An alternative approach is to let $\mathbb{G}$ be the extended real line $\overline{\mathbb{R}}=[-\infty, \infty]$ with the reversed order and conventional addition and multiplication operations. In this case, $f(x)=-x$. We briefly recall three univariate risk measures: the value at risk, which is the most widely used risk measure, and two coherent risk measures, the expected shortfall, and the expected minimum.

The value at risk is defined as the amount of extra capital that a firm needs in order to reduce the probability of going bankrupt to a fixed threshold $\alpha$. It is the opposite of the $\alpha$-quantile of a random variable $X$, i.e.,

$$
\mathrm{V} @ \mathrm{R}_{\alpha}(X)=-\inf \{x: \mathbf{P}\{X \leq x\}>\alpha\}=-F_{X}^{-1}(\alpha)
$$


where $F_{X}$ is the cumulative distribution function of $X$. It can be shown that the value at risk is a homogeneous risk measure, but not a coherent one. It satisfies properties R1, R2, and R3, but not necessarily R4.

The expected shortfall is a coherent risk measure defined as

$$
\operatorname{ES}_{\alpha}(X)=-\frac{1}{\alpha} \int_{0}^{\alpha} F_{X}^{-1}(t) \mathrm{d} t
$$

where $\alpha \in(0,1]$.

The expected minimum is another coherent risk measure defined as

$$
\operatorname{EM}_{1 / n}(X)=-\mathbf{E} \min \left\{X_{1}, X_{2}, \ldots, X_{n}\right\}
$$

where $X_{1}, X_{2}, \ldots, X_{n}$ are independent copies of $X$. The expected minimum belongs to the family of weighted V@Rs and is called Alpha-V@R in [7].

In the following we often consider the Euclidean space $\mathbb{R}^{d}$ extended by adding to it the top and bottom elements at infinity, so that the space then becomes a complete lattice. In order to simplify the notation, we retain the notation $\mathbb{R}^{d}$ for this extended space and $\mathbb{R}$ for the extended real line.

Example 2.6 (Marginalised multivariate vector-valued risk measures) Let $\mathbb{G}$ be $\mathbb{R}^{d}$ with the usual addition, multiplication by positive numbers, and the reversed coordinatewise order, i.e., $a \preceq b$ if $b \leq_{K} a$ with $K=\mathbb{R}_{+}^{d}$. Given a $d$-dimensional random vector $X=\left(X_{1}, \ldots, X_{d}\right)$, any of the aforementioned univariate risk measures $\rho$ yields a risk measure $\varrho(X)=\left(\rho\left(X_{1}\right), \ldots, \rho\left(X_{d}\right)\right)$ with values in $\mathbb{R}^{d}$. In this case, $f(x)=-x$.

\section{Acceptance cones and acceptance sets}

The concept of an acceptance set is the dual one to the risk measure, see $[2,11,16]$. The main idea is that a portfolio $X$ is acceptable if $\varrho(X)$ belongs to a certain subcone $\mathbb{A} \subset \mathbb{G}$ called the acceptance cone. The classical setting (see Example 2.5) corresponds to $\mathbb{G}=\mathbb{R}$ with the reversed order and $\mathbb{A}=(-\infty, 0]$. Every acceptance cone $\mathbb{A}$ is upper with respect to $\preceq$, i.e., if $a \preceq b$ and $a \in \mathbb{A}$, then $b \in \mathbb{A}$. We also assume that

$$
\{a \in \mathbb{G}: \mathbf{e} \preceq a\}=\mathbb{A},
$$

i.e., a deterministic portfolio $x$ is acceptable if and only if $0 \leq_{K} x$.

Given the risk measure $\varrho$, the set $\mathcal{A} \subset L_{d}^{\infty}$ of acceptable portfolios (called the acceptance set) is given by

$$
\mathcal{A}=\left\{X \in L_{d}^{\infty}: \varrho(X) \in \mathbb{A}\right\}=\left\{X \in L_{d}^{\infty}: \mathbf{e} \preceq \varrho(X)\right\} .
$$

If $\varrho$ is coherent, then $\mathcal{A}$ is a cone in $L_{d}^{\infty}$. From $\mathrm{R} 1$ it follows that

$$
\{y: X-y \in \mathcal{A}\}=\{y: \varrho(X-y) \in \mathbb{A}\}=\{y: \varrho(X) \oplus f(-y) \in \mathbb{A}\} .
$$


The $f$-image of the set on the right-hand side is

$$
\begin{aligned}
\varrho_{\mathcal{A}}(X) & =\left\{f(y): y \in \mathbb{R}^{d}, \varrho(X) \oplus f(-y) \in \mathbb{A}\right\} \\
& =\{a \in \mathbb{F}: \varrho(X) \in \mathbb{A} \oplus a\} \\
& =\{a \in \mathbb{F}: a \preceq \varrho(X)\} .
\end{aligned}
$$

Indeed, since the family $\mathbb{F}$ of values of $f$ is a linear space, $\mathbb{A} \oplus a=\{b \oplus a: b \in \mathbb{A}\}$ coincides with the set $\{b \in \mathbb{G}: a \preceq b\}$ for any $a \in \mathbb{F}$.

Note that $\varrho_{\mathcal{A}}(X)$ is not necessarily an element of $\mathbb{G}$, since it may consist of several elements of $\mathbb{G}$. For instance, in Example 2.5 (with $\mathbb{G}=\mathbb{R}$ ), $\varrho_{\mathcal{A}}(X)$ is the set $[\rho(X), \infty)$, while the risk of $X$ is a real number. In this case, one can retrieve the risk of $X$ by taking the infimum of all members of $\varrho_{\mathcal{A}}(X)$. This minimum corresponds to the $\vee$-operation in $\mathbb{R}$ with the reversed order. The following easy observation generalizes the well-known relationship between risk measures and acceptance sets [2,9].

Proposition 3.1 If $\mathbb{F}$ is sup-generating (see [13, p. 28]), i.e.,

$$
b=\bigvee\{a \in \mathbb{F}: a \preceq b\} \quad \text { for all } b \in \mathbb{G},
$$

then

$$
\varrho(X)=\bigvee \varrho_{\mathcal{A}}(X)
$$

In the multivariate case, one often needs the concept of the rejection cone $\mathbb{A}^{\mathrm{r}}=\{a \in \mathbb{G}: a \preceq \mathbf{e}\}$ and rejection set

$$
\mathcal{A}^{\mathrm{r}}=\left\{X \in L_{d}^{\infty}: \varrho(X) \preceq \mathbf{e}\right\} .
$$

While $\mathbb{A}^{\mathrm{r}}$ is a subcone of $\mathbb{G}$, the set $\mathcal{A}^{\mathrm{r}}$ is not necessarily convex even if $\varrho$ is coherent. Indeed, if $X, Y \in \mathcal{A}^{\mathrm{r}}$, then $\varrho(X)+\varrho(Y) \preceq \mathbf{e}$, while R4 no longer suffices to deduce that $\varrho(X+Y) \preceq \mathbf{e}$.

Example 3.2 (Set-valued risk measures) Let $\mathbb{G}_{K}$ be the cone of convex closed sets described in Example 2.4 and $f(x)=-x+K$, so that $\mathbb{F}=\left\{y+K: y \in \mathbb{R}^{d}\right\}$. If $\mathbb{A}=\left\{A \in \mathbb{G}_{K}: K \subseteq A\right\}$, then $\mathbb{F}$ is sup-generating, since for any $F \in \mathbb{G}_{K}$ we have

$$
\bigvee\{a \in \mathbb{F}: a \preceq b\}=\bigcup\left\{y+K: y \in \mathbb{R}^{d},(y+K) \subseteq F\right\}=F .
$$

As in [16, Sect. 2.5], it is possible to choose another acceptance cone $\mathbb{A}^{\prime}$ which is richer than the cone $\mathbb{A}$ defined above. Furthermore, the sup-generating property (3.2) corresponds to the self-consistency property from [16, Property 3.4].

Example 3.3 (Alternative construction of set-valued risk measures) There is also an alternative way to introduce set-valued risk measures. Let $\mathbb{G}_{K}^{\mathrm{r}}$ be the family of complements to the interiors of sets from $\mathbb{G}_{K}$ with the addition operation induced by one from $\mathbb{G}_{K}$, i.e., $F_{1} \oplus F_{2}$ is the complement to the Minkowski sum of the complements to $F_{1}$ and $F_{2}$. The neutral element $\mathbf{e}=K^{\mathrm{r}}$ is then the complement to the 
interior of $K$. If $\mathbb{G}_{K}^{\mathrm{r}}$ is equipped with the inclusion order, then the same arguments as in Example 2.4 confirm that $\mathbb{G}_{K}^{\mathrm{r}}$ is a complete lattice.

If $f(x)=x+K^{\mathrm{r}}, x \in \mathbb{R}^{d}$, then the corresponding family $\mathbb{F}$ is inf-generating (see $[13$, p. 28]), i.e.,

$$
b=\bigwedge\{a \in \mathbb{F}: b \preceq a\} \quad \text { for all } b \in \mathbb{G} .
$$

In this case,

$$
\varrho(X)=\bigwedge \varrho_{\mathcal{A}}(X)
$$

where $\varrho \mathcal{A}^{\mathrm{r}}(X)$ is the $f$-image of all $y \in \mathbb{R}^{d}$ such that $X-y \in \mathcal{A}^{\mathrm{r}}$.

Example 3.4 (Vector-valued risk measures from scalar portfolios) Consider a risk measure $\varrho$ defined on $L_{1}^{\infty}$ with values in $\mathbb{G}=\mathbb{R}^{2}$ with the usual summation and multiplication by scalars and the reversed coordinatewise ordering, i.e., the reversed ordering generated by $K=\mathbb{R}_{+}^{2}$. Such a risk measure may be defined as a vector composed of several univariate risk measures from Example 2.5. In this case, $f(x)=$ $(-x,-x)$, so that $\mathbb{F}$ is the diagonal in $\mathbb{R}^{2}$, which is clearly not sup-generating.

This example explains, by the way, why in the framework of [16] only risk measures that do not increase the dimension of the portfolios have been studied.

\section{Constructions of risk measures}

\subsection{Minimization}

Consider a family $\varrho_{i}, i \in I$, of risk measures on the same cone $\mathbb{G}$, all associated with the same function $f$. Then $\varrho=\bigwedge_{i \in I} \varrho_{i}$ is also a risk measure associated with $f$. If all $\varrho_{i}$ are coherent (resp. homogeneous or convex), the resulting risk measure is coherent (resp. homogeneous or convex). The acceptance set associated with $\varrho$ is the intersection of the acceptance sets of the risk measures $\varrho_{i}, i \in I$.

Example 4.1 (Minimization of univariate risk measures) While it is not interesting to take the minimum of, say, the expected shortfalls at different levels, it is possible to combine members from different families of univariate risk measures. For instance, if $n \geq 1$ and $\alpha \in(0,1]$, then $\max \left\{\mathrm{EM}_{1 / n}(X), \mathrm{ES}_{\alpha}(X)\right\}$ is a coherent risk measure associated with $f(x)=-x$. Note that the maximum of two risk measures corresponds to the minimum in $\mathbb{G}=\mathbb{R}$ with the reversed order.

\subsection{Re-centering}

All random vectors from $L_{d}^{\infty}$ can be naturally centered by subtracting their expected values. This makes it possible to define a risk measure on centered random vectors and then use R1 to extend it onto the whole $L_{d}^{\infty}$. If $\varrho$ is defined on the family of essentially bounded random vectors with mean zero, then the re-centered risk measure is given by

$$
\varrho_{\mathrm{o}}(X)=\varrho(X-\mathbf{E} X) \oplus f(\mathbf{E} X), \quad X \in L_{d}^{\infty}
$$


If $\mathbb{G}$ is $\mathbb{R}^{d}$ or a family of subsets of $\mathbb{R}^{d}$, we rely on the canonical choice of the translation by setting $\varrho_{\mathrm{o}}(X)=\varrho(X-\mathbf{E} X)-\mathbf{E} X$.

It should be noted that $\mathrm{R} 2$ does not hold automatically for re-centered risk measures and has to be checked every time the re-centering is applied.

\subsection{Homogenization}

If $\varrho$ satisfies R1 and R2, it is possible to construct a homogeneous risk measure from it by setting

$$
\varrho_{\mathrm{h}}(X)=\bigwedge_{t>0} \frac{1}{t} \odot \varrho(t X) .
$$

Note that the infimum operation $\bigwedge$ in $\mathbb{G}$ makes sense, since $\mathbb{G}$ is a complete lattice. It is easy to see that $\varrho_{\mathrm{h}}$ satisfies R3. Furthermore, it satisfies R2 and R1 if $f$ is homogeneous. The latter is clearly the case if $f(x)=-x+K, x \in \mathbb{R}^{d}$, for a proper cone $K$, see Example 2.4.

A similar construction produces a translation-invariant risk measure from a general one by

$$
\varrho_{\mathrm{t}}(X)=\bigwedge_{z \in \mathbb{R}^{d}}(\varrho(X+z) \oplus f(-z)) .
$$

Both (4.1) and (4.2) applied together to a function $\varrho$ that satisfies R2 and R4 yield a coherent risk measure.

Example 4.2 If $\mathbb{G}$ is the real line with the reversed order and (4.1) results in a nontrivial function, then $\varrho(t X) \rightarrow 0$ as $t \rightarrow 0$. Similarly, a nontrivial result of (4.2) yields that $\varrho(X+z) \rightarrow-\infty$ as $z \rightarrow \infty$. For instance, these constructions produce trivial results if applied to the risk measure $\mathbf{E}(k-X)_{+}$studied in [14].

\subsection{Worst conditioning}

A single risk measure $\varrho$ can be used to produce a family of risk measures by taking the infimum of the risks associated to the random vectors obtained after certain rearrangements of the underlying probability measure. For each $\alpha \in(0,1]$, define

$$
\varrho_{\alpha}(X)=\bigwedge_{\phi \in \Phi_{\alpha}} \varrho\left(X_{\phi}\right)
$$

where $X_{\phi}=X \circ \phi$, and $\Phi_{\alpha}$ is the family of measurable mappings $\phi: \Omega \mapsto \Omega$ such that $\mathbf{P}\left(\phi^{-1}(A)\right) \leq \alpha^{-1} \mathbf{P}(A)$ for all $A \in \mathfrak{F}$. If $X \in L_{d}^{\infty}$, then $X_{\phi} \in L_{d}^{\infty}$ for any $\alpha \in$ $(0,1]$ and $\phi \in \Phi_{\alpha}$. It is possible to define the worst conditioning alternatively as

$$
\varrho_{\alpha}(X)=\bigwedge_{Y \in \mathcal{P}_{\alpha}(X)} \varrho(Y)
$$

where $\mathcal{P}_{\alpha}(X)$ is the family of all random vectors $Y$ with the property that $\mathbf{P}\{Y \in B\} \leq \alpha^{-1} \mathbf{P}\{X \in B\}$ for all Borel $B \subset \mathbb{R}^{d}$. 
It is easy to show that $\varrho_{\alpha}$ preserves any property that $\varrho$ satisfies from R1-R4. For instance, if $Y \leq_{K} X$ a.s., then $Y_{\phi} \leq_{K} X_{\phi}$ a.s. for any $\phi \in \Phi_{\alpha}$, so that

$$
\varrho_{\alpha}(Y)=\bigwedge_{\phi \in \Phi_{\alpha}} \varrho\left(Y_{\phi}\right) \preceq \bigwedge_{\phi \in \Phi_{\alpha}} \varrho\left(X_{\phi}\right)=\varrho_{\alpha}(X)
$$

whenever $\varrho$ satisfies R2. If $X, Y \in L_{d}^{\infty}$ and $\varrho$ satisfies R4, then

$$
\begin{aligned}
\varrho_{\alpha}(X+Y) & =\bigwedge_{\phi \in \Phi_{\alpha}} \varrho\left((X+Y)_{\phi}\right)=\bigwedge_{\phi \in \Phi_{\alpha}} \varrho\left(X_{\phi}+Y_{\phi}\right) \\
& \succeq \bigwedge_{\phi \in \Phi_{\alpha}}\left(\varrho\left(X_{\phi}\right) \oplus \varrho\left(Y_{\phi}\right)\right) \succeq \bigwedge_{\phi \in \Phi_{\alpha}} \varrho\left(X_{\phi}\right) \oplus \bigwedge_{\phi \in \Phi_{\alpha}} \varrho\left(Y_{\phi}\right) \\
& =\varrho_{\alpha}(X) \oplus \varrho_{\alpha}(Y) .
\end{aligned}
$$

Consider now the setting of univariate risk measures from Example 2.5, i.e., $X$ is a random variable from $L_{1}^{\infty}$, and $\mathbb{G}$ is the real line with the reversed order. The simplest coherent risk measure is the opposite of the expectation of a random variable. In fact, this risk measure appears from the expected shortfall when $\alpha=1$, i.e., $\operatorname{ES}_{1}(X)=$ $-\mathbf{E} X$. The worst conditioning applied to the opposite of the expectation yields

$$
\begin{aligned}
(-\mathbf{E})_{\alpha}(X) & =\sup _{\phi \in \Phi_{\alpha}}\left\{-\mathbf{E}\left(X_{\phi}\right)\right\}=-\inf _{\phi \in \Phi_{\alpha}} \mathbf{E}\left(X_{\phi}\right)=-\inf _{\phi \in \Phi_{\alpha}} \int X(\phi(\omega)) \mathbf{P}(\mathrm{d} \omega) \\
& =-\inf _{\phi \in \Phi_{\alpha}} \int X(\omega) \mathbf{P} \phi^{-1}(\mathrm{~d} \omega)=-\inf _{\phi \in \Phi_{\alpha}} \mathbf{E}_{\mathbf{P} \phi^{-1}} X
\end{aligned}
$$

where $\mathbf{E}_{\mathbf{P} \phi^{-1}}$ denotes the expectation with respect to the probability measure $\mathbf{P} \phi^{-1}$.

In general, $-\inf _{\phi \in \Phi_{\alpha}} \mathbf{E}_{\mathbf{P} \phi^{-1}} X \leq \operatorname{ES}_{\alpha}(X)$ with equality if $(\Omega, \mathfrak{F}, \mathbf{P})$ is nonatomic. Without loss of generality assume that $\Omega=[0,1], \mathbf{P}$ is the Lebesgue measure restricted to $[0,1]$, and $X$ is an increasing mapping from $[0,1]$ into $\mathbb{R}$, which implies that $X(\omega)=F_{X}^{-1}(\omega)$ for all $\omega \in[0,1]$, where $F_{X}$ is the cumulative distribution function of $X$. The infimum of $\mathbf{E}_{\mathbf{P} \phi^{-1}} X$ over all $\phi \in \Phi_{\alpha}$ is achieved when $X \circ \phi$ takes the smallest possible values with the highest possible probabilities, and thus it is attained at $\phi^{\prime}(\omega)=\alpha \omega$. We conclude that

$$
(-\mathbf{E})_{\alpha}(X)=-\int X(\alpha \omega) \mathrm{d} \omega=-\frac{1}{\alpha} \int_{0}^{\alpha} F_{X}^{-1}(t) \mathrm{d} t=\operatorname{ES}_{\alpha}(X),
$$

i.e., the expected shortfall appears by applying the worst conditioning construction to the opposite of the expectation.

Example 4.3 (Worst conditioning of the expected shortfall) Let us now apply the worst conditioning to the expected shortfall at level $\beta$,

$$
\begin{aligned}
\left(\mathrm{ES}_{\beta}\right)_{\alpha}(X) & =\sup _{\phi_{1} \in \Phi_{\alpha}} \mathrm{ES}_{\beta}\left(X_{\phi_{1}}\right)=\sup _{\phi_{1} \in \Phi_{\alpha}}\left(-\inf _{\phi_{2} \in \Phi_{\beta}} \mathbf{E}_{\mathbf{P} \phi_{1}^{-1}} X_{\phi_{2}}\right) \\
& =-\inf _{\phi_{1} \in \Phi_{\alpha}, \phi_{2} \in \Phi_{\beta}} \mathbf{E}_{\mathbf{P} \phi_{1}^{-1} \phi_{2}^{-1}} X .
\end{aligned}
$$


Clearly $\phi_{2} \circ \phi_{1} \in \Phi_{\alpha \beta}$ and thus $\left(\mathrm{ES}_{\beta}\right)_{\alpha}(X) \leq \mathrm{ES}_{\alpha \beta}(X)$. If the probability space is nonatomic, all mappings from $\Phi_{\alpha \beta}$ can be written as the composition of a mapping from $\Phi_{\alpha}$ and a mapping from $\Phi_{\beta}$, so that $\left(\mathrm{ES}_{\beta}\right)_{\alpha}(X)=\mathrm{ES}_{\alpha \beta}(X)$. One can say that the expected shortfall is stable under the worst conditioning.

Example 4.4 (Worst conditioned V@ $\mathrm{R}_{\alpha}$ ) Let us finally apply the worst conditioning construction to the value at risk at level $\beta$ considered on the nonatomic probability space $\Omega=[0,1]$ with $\mathbf{P}$ being the Lebesgue measure. Without loss of generality assume that $X$ is increasing, so that $X(\omega)=F_{X}^{-1}(\omega)$. The infimum below is attained at $\phi^{\prime}(\omega)=\alpha \omega$ and since $X_{\phi^{\prime}}$ is also increasing, we have $X_{\phi^{\prime}}(\omega)=F_{X_{\phi^{\prime}}}^{-1}(\omega)$. Thus

$$
\begin{aligned}
\left(\mathrm{V} @ \mathrm{R}_{\beta}\right)_{\alpha}(X) & =-\inf _{\phi \in \Phi_{\alpha}} F_{X_{\phi}}^{-1}(\beta)=-X_{\phi^{\prime}}(\beta)=-X(\alpha \beta)=-F_{X}^{-1}(\alpha \beta) \\
& =\mathrm{V} @ \mathrm{R}_{\alpha \beta}(X) .
\end{aligned}
$$

\subsection{Transformations of risks}

Risk measures with values in a cone $\mathbb{G}$ can be further transformed by mapping $\mathbb{G}$ into another cone $\mathbb{G}^{\prime}$ using a map $h$. The aim may be to change the dimensionality (cf. [16]) or produce a vector-valued risk measure from a set-valued one. The map $h: \mathbb{G} \mapsto \mathbb{G}^{\prime}$ that transforms any $\mathbb{G}$-valued risk measure $\varrho$ into the $\mathbb{G}^{\prime}$-valued risk measure $h(\varrho(\cdot))$ will be called a risk transformation. If $h$ respects the coherence property of risk measures, it will be called a coherent map.

Let us denote by $\preccurlyeq$ the partial order in $\mathbb{G}^{\prime}$ which we assume to be compatible with the (commutative) addition operation and multiplication by scalars. The additive operation on $\mathbb{G}^{\prime}$ and the multiplication by numbers will also be denoted by $\oplus$ and $\odot$, respectively. In the following result, we list the properties that a coherent map should possess. The mapping that assesses the risk of a deterministic portfolio in the new cone $\mathbb{G}^{\prime}$ will be $h(f(\cdot))$. Recall that $\mathbb{F}$ denotes the family of possible values of the function $f$.

Proposition 4.5 A map $h: \mathbb{G} \mapsto \mathbb{G}^{\prime}$ is a risk transformation if it is:

(i) nondecreasing, i.e., $h(a) \preccurlyeq h(b)$ if $a \preceq b$;

(ii) linear on $\mathbb{F}$, i.e., $h(a \oplus b)=h(a) \oplus h(b)$ for all $b \in \mathbb{G}$ and $a \in \mathbb{F}$.

Further, $h$ is a coherent map if $h$ is homogeneous, i.e., $h(t \odot a)=t \odot h(a)$ for all $t>0$ and $a \in \mathbb{G}$, and also satisfies

$$
h(a) \oplus h(b) \preccurlyeq h(a \oplus b)
$$

for all $a, b \in \mathbb{G}$.

Proof Since $\varrho$ satisfies $\mathrm{R} 1$ and $f(y) \in \mathbb{F}$, we have, for all $y \in \mathbb{R}^{d}$,

$$
h(\varrho(X+y))=h(f(y) \oplus \varrho(X))=h(f(y)) \oplus h(\varrho(X)),
$$


i.e., R1 holds. Property R2 holds because $h$ is nondecreasing. The homogeneity of $h(\varrho(\cdot))$ is evident if $h$ is homogeneous. If $\varrho$ is coherent and (4.3) holds, then

$$
h(\varrho(X)) \oplus h(\varrho(Y)) \preccurlyeq h(\varrho(X) \oplus \varrho(Y)) \preccurlyeq h(\varrho(X+Y)) .
$$

As an immediate consequence of Proposition 4.5, we deduce that every linear nondecreasing map is coherent. Such maps between partially ordered vector spaces are called Riesz homomorphisms, see [19, Sect. 18].

Example 4.6 (Vector-valued risk measures from set-valued ones) A particularly important instance of transformation of risks arises if $\mathbb{G}_{K}$ is the family of convex closed subsets of $\mathbb{R}^{d}$ with inclusion order defined in Example 2.4 and $\mathbb{G}^{\prime}$ is $\mathbb{R}^{d}$ with the reversed $\leq_{K}$-order for a proper Euclidean cone $K$. The reversing is needed, since $y+K \subseteq z+K$ (i.e., $y+K \preceq z+K$ ) if and only if $z \leq_{K} y$.

The cone $K$ is said to be a Riesz cone if $\mathbb{R}^{d}$ with $\leq_{K}$-order is a Riesz space, i.e., for every $x, y \in \mathbb{R}^{d}$ their supremum is well defined. From [19, Th. 26.11] it follows that each Riesz cone can be represented as $K=\left\{u \in \mathbb{R}^{d}: A u \in \mathbb{R}_{+}^{d}\right\}$ for a nonsingular $d \times d$ matrix $A$ with nonnegative entries, i.e., $K=A^{-1} \mathbb{R}_{+}^{d}$. The matrix $A$ can represent possible transfers between the assets so that $Y \leq_{K} X$ if and only if $A Y$ is coordinatewise smaller than $A X$.

Assume that $K$ is a Riesz cone. Then it is easy to see that $\mathbb{G}^{\prime}$ is a complete lattice. Let $h(F)$ denote the supremum of $F \subset \mathbb{R}^{d}$ in $\mathbb{G}^{\prime}$ (i.e., the $\leq_{K}$-infimum of $F$ ). If $\varrho$ is a $\mathbb{G}$-valued risk measure, then $h(\varrho(\cdot))$ is a vector-valued risk measure. Indeed, the map $h$ is monotone and homogeneous. Since

$$
h(F-y+K)=h(F-y)=h(F)-y=h(F)+h(-y+K),
$$

$h$ is linear on $\mathbb{F}$. Finally, $h$ satisfies (4.3), since $x=h\left(F_{1}\right)$ and $y=h\left(F_{2}\right)$ imply that $F_{1}+F_{2} \subseteq\left(x_{1}+x_{2}\right)+K$.

It is also possible to produce vector-valued risk measures from set-valued risk measures in the cone $\mathbb{G}_{K}^{\mathrm{r}}$ from Example 3.3 if $h$ is chosen to be the supremum in $\mathbb{G}^{\prime}$ of the complement of $F \in \mathbb{G}_{K}^{\mathrm{r}}$.

Example 4.7 (Linear transformations of vector-valued risk measures) Let $\varrho$ be a risk measure on $L_{d}^{\infty}$ with values in $\mathbb{R}^{d}$ with the reversed $\leq_{K}$-order for a Riesz cone $K=A^{-1} \mathbb{R}_{+}^{d}$. Note that $K$ generates the order both on $L_{d}^{\infty}$ and on the space of values for $\varrho$. Then

$$
\varrho(X)=A^{-1} \tilde{\varrho}(A X)
$$

where $\tilde{\varrho}$ is a risk measure with values in $\mathbb{R}^{d}$ with the reversed coordinatewise order.

Example 4.8 (Scalar risk measures from vector-valued ones) Let $K$ be a Riesz cone and $\mathbb{G}=\mathbb{R}^{d}$ with the reversed $\leq_{K}$-order. Define $\mathbb{G}^{\prime}=\mathbb{R}$ with the reversed natural order. Finally, let $h(a)=\langle a, u\rangle$, where $\langle\cdot, \cdot\rangle$ is the scalar product and $u$ belongs to the positive dual cone to $K$, i.e., $\langle u, v\rangle \geq 0$ for all $v \in K$. Clearly $h$ is a coherent map, and we obtain univariate risk measures as those of Example 2.5, but now for multivariate portfolios. 


\section{Depth-trimmed regions}

Depth functions assign to a point its degree of centrality with respect to the distribution of a random vector, see [24]. The higher the depth of a point is, the more central this point is with respect to the distribution of the random vector. Depth-trimmed (or central) regions are sets of central points associated with a random vector. Given a depth function, depth-trimmed regions can be obtained as its level sets. With a $d$-dimensional random vector $X$ we associate the family of depth-trimmed regions, i.e., sets $\mathrm{D}^{\alpha}(X), \alpha \in(0,1]$, such that the following properties hold for all $\alpha \in[0,1]$ and all $X \in L_{d}^{\infty}$ :

D1 $\mathrm{D}^{\alpha}(X+y)=\mathrm{D}^{\alpha}(X)+y$ for all $y \in \mathbb{R}^{d}$;

$\mathrm{D} 2 \mathrm{D}^{\alpha}(t X)=t \mathrm{D}^{\alpha}(X)$ for all $t>0$;

D3 $\mathrm{D}^{\alpha}(X) \subseteq \mathrm{D}^{\beta}(X)$ if $\alpha \geq \beta$;

D4 $\mathrm{D}^{\alpha}(X)$ is connected and closed.

Note that the addition of $y$ in D1 and the multiplication by $t$ in D2 are the conventional translation and the rescaling of sets in $\mathbb{R}^{d}$.

These properties are similar to those discussed by Zuo and Serfling [25, Theorem 3.1]. Additionally, [25] requires that the depth-trimmed regions are invariant with respect to linear transformations, i.e., $\mathrm{D}^{\alpha}(A X)=A \mathrm{D}^{\alpha}(X)$ for any nonsingular matrix $A$.

We consider two additional properties of depth-trimmed regions that, to our knowledge, have not been studied in the literature so far:

D5 if $Y \leq_{K} X$ a.s., then $\mathrm{D}^{\alpha}(X) \subseteq \mathrm{D}^{\alpha}(Y) \oplus K$ and $0 \in \mathrm{D}^{\alpha}(X) \subseteq K$ if $X=0$ a.s.; D6 $\mathrm{D}^{\alpha}(X+Y) \subseteq \mathrm{D}^{\alpha}(X) \oplus \mathrm{D}^{\alpha}(Y)$.

Observe that depth-trimmed regions are closed subsets of $\mathbb{R}^{d}$ and the addition operation in D5 and D6 is the closed Minkowski addition. Later on we shall see that D6 is closely related to the coherence property of risk measures.

Example 5.1 (Halfspace trimming) The halfspace trimmed regions are built as the intersections of closed halfspaces whose probabilities are not smaller than a given value:

$$
\operatorname{HD}^{\alpha}(X)=\bigcap\{H: H \text { closed halfspace with } \mathbf{P}\{X \in H\} \geq 1-\alpha\} .
$$

The above definition of the halfspace trimmed regions is taken from Massé and Theodorescu [20]. Alternatively, the nonstrict inequality in the definition of $\mathrm{HD}^{\alpha}$ could be replaced by the strict one, see [23]. However, the definition of [20] leads to a simpler relationship between the value at risk and the univariate halfspace trimming, see Sect. 6.

It is well known that the halfspace trimmed regions satisfy D1-D4 and are compact and convex. The new property D6 does not hold in general; this can be shown in the univariate case, using examples for which the value at risk does not satisfy R4. The monotonicity property D5 does not hold in general either. 
However, it is possible to build a variant of the halfspace trimmed regions satisfying D5. We define the monotone halfspace trimmed regions as

$$
\operatorname{HD}_{K}^{\alpha}(X)=\bigcap_{u \in K^{*}}\left\{H_{u}: \mathbf{P}\left\{X \in H_{u}\right\} \geq 1-\alpha\right\},
$$

where $H_{u}=\left\{x \in \mathbb{R}^{d}:\langle x, u\rangle \geq 1\right\}$ is a halfspace, and $K^{*}=\{u:\langle u, v\rangle \geq 0, v \in K\}$ is the positive dual cone to $K$. The monotone halfspace trimmed regions satisfy D1-D5 and are nonempty for all $\alpha \in(0,1]$.

Example 5.2 (Zonoid trimming) Koshevoy and Mosler [18] defined zonoid trimmed regions for an integrable random vector $X$ in $\mathbb{R}^{d}$ as

$$
\mathrm{ZD}^{\alpha}(X)=\left\{\mathbf{E}[X \ell(X)]: \ell: \mathbb{R}^{d} \mapsto\left[0, \alpha^{-1}\right] \text { measurable and } \mathbf{E} \ell(X)=1\right\}
$$

where $\alpha \in(0,1]$. Properties D1-D4 together with the convexity and boundedness (and thus compactness) are already derived in [18]. The proofs of D5 and D6 do not involve serious technical difficulties.

Example 5.3 (Expected convex hull trimming) Expected convex hull regions of a random vector $X$ at level $n^{-1}$ for $n \geq 1$ are defined by Cascos [5] as the selection (Aumann) expectation of the convex hull of $n$ independent copies $X_{1}, \ldots, X_{n}$ of $X$; see [21, Sect. 2.1] for the definition of expectation for random sets. The expected convex hull region can be given implicitly in terms of its support function as

$$
h\left(\mathrm{CD}^{1 / n}(X), u\right)=\mathbf{E} \max \left\{\left\langle X_{1}, u\right\rangle,\left\langle X_{2}, u\right\rangle, \ldots,\left\langle X_{n}, u\right\rangle\right\} \quad \text { for all } u \in \mathbb{R}^{d}
$$

where $\langle\cdot, \cdot\rangle$ is the scalar product. Note that for any $F \subset \mathbb{R}^{d}$ its support function is given by $h(F, u)=\sup \{\langle x, u\rangle: x \in F\}$ for $u \in \mathbb{R}^{d}$. The expected convex hull regions satisfy properties D1-D6 and are compact and convex.

Example 5.4 (Integral trimming) Let $\mathcal{F}$ be a family of measurable functions from $\mathbb{R}^{d}$ into $\mathbb{R}$. Cascos and López-Díaz [6] defined the family of integral trimmed regions as

$$
\begin{aligned}
\mathrm{D}_{\mathcal{F}}^{\alpha}(X) & =\bigcup_{Y \in \mathcal{P}_{\alpha}(X)}\left\{x \in \mathbb{R}^{d}: \mathfrak{f}(x) \leq \mathbf{E} \mathfrak{f}(Y) \text { for all } \mathfrak{f} \in \mathcal{F}\right\} \\
& =\bigcup_{Y \in \mathcal{P}_{\alpha}(X)} \bigcap_{\mathfrak{f} \in \mathcal{F}} \mathfrak{f}^{-1}((-\infty, \mathbf{E} \mathfrak{f}(Y)])
\end{aligned}
$$

where $\mathcal{P}_{\alpha}(X)$ is defined in Sect. 4.4. All families of integral trimmed regions satisfy D3. Other properties of the integral trimmed regions heavily depend on their generating family of functions. For instance, if, for any $\mathfrak{f} \in \mathcal{F}, t>0$, and $z \in \mathbb{R}^{d}$, the function $\mathfrak{f}_{t, z}$ defined as $\mathfrak{f}_{t, z}(x)=\mathfrak{f}(t x+z)$ belongs to $\mathcal{F}$, then the integral trimmed regions generated by $\mathcal{F}$ satisfy properties D1 and D2. 
If $\mathcal{F}=\left\{\mathfrak{f}_{t, z}: t>0, z \in \mathbb{R}^{d}\right\}$ with a continuous and $\leq_{K}$-decreasing function $\mathfrak{f}$, then

$$
\mathrm{D}_{\mathcal{F}}^{\alpha}(X)=\bigcup_{Y \in \mathcal{P}_{\alpha}(X)} \bigcap_{t>0, z \in \mathbb{R}^{d}}\left[\frac{1}{t}\left(\mathfrak{f}^{-1}(\mathbf{E} \mathfrak{f}(t Y+z))-z\right) \oplus K\right] .
$$

Hereafter we assume that all depth-trimmed regions satisfy D1-D5.

\section{Risk measures generated by depth-trimmed regions}

As a motivation for the following, note that, for an essentially bounded random variable $X, \alpha \in(0,1]$ and $n \geq 1$, we have

$$
\begin{aligned}
\mathrm{V} @ \mathrm{R}_{\alpha}(X) & =-\min \mathrm{HD}_{[0, \infty)}^{\alpha}(X), \\
\mathrm{ES}_{\alpha}(X) & =-\min \mathrm{ZD}^{\alpha}(X), \\
\mathrm{EM}_{1 / n}(X) & =-\min \mathrm{CD}^{1 / n}(X) .
\end{aligned}
$$

The following example provides another argument showing relationships between depth-trimmed regions and risk measures.

Example 6.1 (Depth-trimmed regions as set-valued risk measures) Observe that any depth-trimmed region that satisfies D1-D5 can be transformed into a set-valued risk measure from Definition 2.3. Namely, $\varrho(X)=\mathrm{D}^{\alpha}(X) \oplus K$ is a risk measure in the cone $\mathbb{G}_{K}$ of closed subsets of $\mathbb{R}^{d}$ with the addition operation being the closed Minkowski addition and the reversed inclusion order. Because of the reversed order, the function $f$ is given by $f(x)=x+K$. However, the obtained risk measure is not coherent even if D6 holds.

In order to construct a coherent risk measure from depth-trimmed regions, define $\varrho(X)$ to be the closure of the complement to $\mathrm{D}^{\alpha}(X) \oplus K$. Then $\varrho$ becomes a coherent risk measure in the cone $\mathbb{G}_{K}^{\mathrm{r}}$ from Example 3.3 if the depth-trimmed region satisfies D1-D6.

In general, a random portfolio $X$ will be acceptable or not depending on the depthtrimmed region of level $\alpha$ associated with $X$. Since the depth-trimmed regions are subsets of the space $\mathbb{R}^{d}$ where $X$ takes its values, we need to map it into the space $\mathbb{G}$ where risk measures take their values. This map is provided by the function $f$ from Definition 2.3. Then

$$
\mathbb{D}^{\alpha}(X)=f\left(\mathrm{D}^{\alpha}(X) \oplus K\right)
$$

is a subset of $\mathbb{G}$. Recall that the acceptance cone $\mathbb{A}$ is a subset of $\mathbb{G}$ that characterizes the acceptable values of the risk measure, see (3.1).

Definition 6.2 The acceptance set at level $\alpha$ associated with the depth-trimmed region $\mathrm{D}^{\alpha}(\cdot)$ and function $f$ is defined as

$$
\mathcal{A}_{\alpha}=\left\{X \in L_{d}^{\infty}: \mathbb{D}^{\alpha}(X) \subseteq \mathbb{A}\right\} .
$$


Theorem 6.3 The acceptance sets associated with depth-trimmed regions satisfy the following properties:

(i) $0 \in \mathcal{A}_{\alpha}$ for all $\alpha$;

(ii) if $\alpha \geq \beta$, then $\mathcal{A}_{\beta} \subseteq \mathcal{A}_{\alpha}$;

(iii) if $X \in \mathcal{A}_{\alpha}$, then $t X \in \mathcal{A}_{\alpha}$ for all $t>0$;

(iv) if $X \in \mathcal{A}_{\alpha}$ and $f(x) \in \mathbb{A}$, then $x+X \in \mathcal{A}_{\alpha}$;

(v) if $Y \in \mathcal{A}_{\alpha}$ and $Y \leq_{K} X$ a.s., then $X \in \mathcal{A}_{\alpha}$;

(vi) if $X, Y \in \mathcal{A}_{\alpha}$ and D6 holds, then $X+Y \in \mathcal{A}_{\alpha}$.

Proof (i) By D5, $\mathbb{D}^{\alpha}(0)=f(K) \subseteq \mathbb{A}$, i.e., $0 \in \mathcal{A}_{\alpha}$ for all $\alpha$.

(ii) By $\mathrm{D} 3, \mathrm{D}^{\alpha}(X) \subseteq \mathrm{D}^{\beta}(X)$ whenever $\alpha \geq \beta$. Thus $\mathbb{D}^{\alpha}(X) \subseteq \mathbb{D}^{\beta}(X)$ and $\mathcal{A}_{\beta} \subseteq \mathcal{A}_{\alpha}$ trivially holds.

(iii) By D2 and the homogeneity of $f$, we have $\mathbb{D}^{\alpha}(t X)=t \odot \mathbb{D}^{\alpha}(X)$ for all $t>0$. Since $\mathbb{A}$ is a cone, $\mathbb{D}^{\alpha}(t X) \subseteq \mathbb{A}$ if and only if $\mathbb{D}^{\alpha}(X) \subseteq \mathbb{A}$.

(iv) Let $f(x) \in \mathbb{A}$. By D1 and (2.1), we have $\mathbb{D}^{\alpha}(X+x)=f\left(\mathrm{D}^{\alpha}(X)+x \oplus K\right)=$ $\mathbb{D}^{\alpha}(X)+f(x) \subseteq \mathbb{A}$ because $\mathbb{A}$ is a (convex) cone. By (6.1), $X+x \in \mathcal{A}_{\alpha}$.

(v) Note that $f\left(\mathrm{D}^{\alpha}(Y) \oplus K\right) \subseteq \mathbb{A}$. By D5, $\mathrm{D}^{\alpha}(X) \oplus K \subseteq \mathrm{D}^{\alpha}(Y) \oplus K$ and thus $f\left(\mathrm{D}^{\alpha}(X) \oplus K\right) \subseteq \mathbb{A}$.

(vi) By (2.1) and D6,

$$
\mathbb{D}^{\alpha}(X+Y) \subseteq f\left(\mathrm{D}^{\alpha}(X) \oplus \mathrm{D}^{\alpha}(Y) \oplus K\right)=\mathbb{D}^{\alpha}(X) \oplus \mathbb{D}^{\alpha}(Y) \subseteq \mathbb{A} .
$$

Finally, the fact that $\mathbb{A}$ is a convex cone yields that $X+Y \in \mathcal{A}_{\alpha}$.

Similarly to the construction used in Sect. 3, we measure the risk of a portfolio $X$ in terms of the collection of deterministic portfolios $x$ that cancel the risk induced by $X$ and make $X+x$ acceptable.

Definition 6.4 The risk measure induced by a family of depth-trimmed regions $\mathrm{D}^{\alpha}$ at level $\alpha$ is given by

$$
s_{\alpha}(X)=\bigvee\left\{f(y): f\left(\mathrm{D}^{\alpha}(X-y) \oplus K\right) \subseteq \mathbb{A}, y \in \mathbb{R}^{d}\right\}
$$

By D1, $s_{\alpha}(X)$ can be given alternatively in terms of the acceptance set at level $\alpha$ as

$$
s_{\alpha}(X)=\bigvee\left\{f(y): X-y \in \mathcal{A}_{\alpha}, y \in \mathbb{R}^{d}\right\}
$$

Theorem 6.5 Assume that $\mathbb{F}$ is sup-generating. Then the mapping $s_{\alpha}(X)$ satisfies

$$
s_{\alpha}(X)=\bigwedge \mathbb{D}^{\alpha}(X)
$$

and so becomes a homogeneous risk measure associated with $f$. If the family of depth-trimmed regions satisfies D6, then $s_{\alpha}(X)$ is a coherent risk measure such that $s_{\alpha}(X) \succeq s_{\beta}(X)$ for $\alpha \geq \beta$. 
Proof The linearity of $f$ and (6.2) imply that

$$
\begin{aligned}
s_{\alpha}(X) & =\bigvee\left\{f(y): f\left(\mathrm{D}^{\alpha}(X) \oplus K\right) \subseteq \mathbb{A} \oplus f(y)\right\} \\
& =\bigvee\left\{f(y): c \in \mathbb{A} \oplus f(y) \text { for all } c \in \mathbb{D}^{\alpha}(X)\right\} \\
& =\bigvee\left\{f(y): f(y) \preceq c \text { for all } c \in \mathbb{D}^{\alpha}(X)\right\} \\
& =\bigvee\left\{a \in \mathbb{F}: a \preceq \bigwedge \mathbb{D}^{\alpha}(X)\right\},
\end{aligned}
$$

so that (6.4) follows from the sup-generating property (3.2).

If $X=x$ a.s., then $s_{\alpha}(X)=\bigwedge f\left(x+\mathrm{D}^{\alpha}(0) \oplus K\right)=f(x)$, since $\bigwedge f\left(\mathrm{D}^{\alpha}(0) \oplus K\right)$ $=\mathbf{e}$ by D5 and $f$ is nondecreasing. By (2.1) and D1, we deduce that $s_{\alpha}(X) \oplus f(y)$ $=s_{\alpha}(X+y)$, so R1 holds.

If $Y \leq_{K} X$ a.s., then $\mathrm{D}^{\alpha}(X) \subseteq \mathrm{D}^{\alpha}(Y) \oplus K$ by D5. Thus R2 holds, since

$$
s_{\alpha}(Y)=\bigwedge f\left(\mathrm{D}^{\alpha}(Y) \oplus K\right) \preceq \bigwedge f\left(\mathrm{D}^{\alpha}(X) \oplus K\right)=s_{\alpha}(X) .
$$

Property $\mathrm{R} 3$ follows directly from $\mathrm{D} 2$, the fact that $K$ is a cone, and the homogeneity of $f$. If D6 holds, then $\mathrm{D}^{\alpha}(X+Y) \oplus K \subseteq\left(\mathrm{D}^{\alpha}(X) \oplus K\right) \oplus\left(\mathrm{D}^{\alpha}(Y) \oplus K\right)$, whence

$$
s_{\alpha}(X+Y)=\bigwedge \mathbb{D}^{\alpha}(X+Y) \succeq \bigwedge \mathbb{D}^{\alpha}(X) \oplus \bigwedge \mathbb{D}^{\alpha}(Y)=s_{\alpha}(X) \oplus s_{\alpha}(X),
$$

i.e., R4 holds. Finally, the ordering of the risks with respect to $\alpha$ follows from D3.

Now we describe a dual construction, based on rejection sets, of set-valued risk measures associated with depth-trimmed regions. The rejection set at level $\alpha$ associated with $\mathrm{D}^{\alpha}(\cdot)$ is given by

$$
\mathcal{A}_{\alpha}^{\mathrm{r}}=\left\{X \in L_{d}^{\infty}: \mathbb{D}^{\alpha}(X) \cap \mathbb{A}^{\mathrm{r}} \neq \emptyset\right\}=\left\{X \in L_{d}^{\infty}: \mathrm{D}^{\alpha}(X) \cap(-K) \neq \emptyset\right\} .
$$

Assuming that $\mathbb{F}$ is inf-generating, by (3.3) we have

$$
s_{\alpha}^{\mathrm{r}}(X)=\bigwedge \varrho_{\mathcal{A}_{\alpha}^{\mathrm{r}}}(X),
$$

where $s_{\alpha}^{\mathrm{r}}(X)$ is also given by (6.3) with $\mathcal{A}_{\alpha}$ replaced by $\mathcal{A}_{\alpha}^{\mathrm{r}}$. It is possible to reproduce Theorem 6.5 in this dual framework and obtain that

$$
s_{\alpha}^{\mathrm{r}}(X)=\bigwedge \mathbb{D}^{\alpha}(X) .
$$

Further, $s_{\alpha}^{r}$ is a homogeneous risk measure which is also coherent if D6 holds.

Example 6.6 (Set-valued risk measures from depth-trimmed regions) In the setting of Example 3.2, $f(x)=-x+K$, so that Theorem 6.5 implies that

$$
\begin{aligned}
s_{\alpha}(X) & =\bigcap_{x \in \mathrm{D}^{\alpha}(X)}(-x+K)=\bigcap_{x \in \mathrm{D}^{\alpha}(X)}\left\{z \in \mathbb{R}^{d}:-x \leq_{K} z\right\} \\
& =\left\{z \in \mathbb{R}^{d}: z+\mathrm{D}^{\alpha}(X) \subseteq K\right\}=\left\{z \in \mathbb{R}^{d}: \mathrm{D}^{\alpha}(X) \subseteq(-z+K)\right\} .
\end{aligned}
$$


If $K$ is a Riesz cone, then there exists the infimum of $\mathrm{D}^{\alpha}(X)$ with respect to the $\leq_{K}$-order (denoted as $\left.\wedge_{K} \mathrm{D}^{\alpha}(X)\right)$, so that

$$
s_{\alpha}(X)=\bigcap_{x \in \mathrm{D}^{\alpha}(X)}\left\{z \in \mathbb{R}^{d}:-z \leq_{K} x\right\}=-\wedge_{K} \mathrm{D}^{\alpha}(X)+K .
$$

Therefore, risk measures generated by depth-trimmed regions using the acceptance cone are not particularly interesting, since they are essentially vector-valued. In Example 9.1 it will be shown that vector-valued risk measures are necessarily marginalized, i.e., they appear from the scheme of Example 2.6.

However, the rejection construction produces more interesting set-valued risk measures. Namely, in the setting of Example 3.3 with $f(x)=x+K^{\mathrm{r}}$, the corresponding risk measure $s_{\alpha}^{\mathrm{r}}(X)$ is the closure to the complement of $\mathrm{D}^{\alpha}(X) \oplus K$. The obtained risk measure takes values in the cone $\mathbb{G}_{K}^{\mathrm{r}}$.

\section{Basic risk measures associated with depth-trimmed regions}

Let us now specialize the constructions from Sect. 6 for $X=\left(X_{1}, X_{2}, \ldots, X_{d}\right) \in L_{d}^{\infty}$ and several basic definitions of depth-trimmed regions and set-valued risk measures with values either in $\mathbb{G}_{K}$ or $\mathbb{G}_{K}^{\mathrm{r}}$ with a Riesz cone $K$. Recall that set-valued risk measures with values in $\mathbb{G}_{K}$ can be represented as $x+K$ for some $x \in \mathbb{R}^{d}$, i.e., are effectively vector-valued. Similar constructions are possible for $\mathbb{G}_{K}^{\mathrm{r}}$-valued risk measures. In this case, the $\leq_{K}$-infimum of the complement to $\mathbb{G}_{K}^{\mathrm{r}}$-valued risk measures also yields a vector-valued risk measure, see Example 4.6.

Risk measures generated by monotone halfspace trimming The monotone halfspace trimming induces a homogeneous risk measure, i.e., R3 holds. This set-valued risk measure is given by $s_{\alpha}\left(X_{1}\right)=\left[\mathrm{V} @ \mathrm{R}_{\alpha}\left(X_{1}\right),+\infty\right)$ in the univariate case. In general,

$$
\begin{aligned}
& s_{\alpha}\left(X_{1}, X_{1}, \ldots, X_{1}\right)=\left(\mathrm{V} @ \mathrm{R}_{\alpha}\left(X_{1}\right), \mathrm{V} @ \mathrm{R}_{\alpha}\left(X_{1}\right), \ldots, \mathrm{V} @ \mathrm{R}_{\alpha}\left(X_{1}\right)\right)+K, \\
& s_{\alpha}(X) \supseteq\left(\mathrm{V} @ \mathrm{R}_{\alpha}\left(X_{1}\right), \mathrm{V} @ \mathrm{R}_{\alpha}\left(X_{2}\right), \ldots, \mathrm{V} @ \mathrm{R}_{\alpha}\left(X_{d}\right)\right)+K .
\end{aligned}
$$

Risk measures generated by zonoid trimming The zonoid trimming induces coherent risk measures. Then $s_{\alpha}\left(X_{1}\right)=\left[\mathrm{ES}_{\alpha}\left(X_{1}\right),+\infty\right)$ and, in the multivariate setting,

$$
\begin{array}{r}
s_{\alpha}\left(X_{1}, X_{1}, \ldots, X_{1}\right)=\left(\mathrm{ES}_{\alpha}\left(X_{1}\right), \mathrm{ES}_{\alpha}\left(X_{1}\right), \ldots, \mathrm{ES}_{\alpha}\left(X_{1}\right)\right)+K, \\
s_{\alpha}(X) \supseteq\left(\mathrm{ES}_{\alpha}\left(X_{1}\right), \mathrm{ES}_{\alpha}\left(X_{2}\right), \ldots, \mathrm{ES}_{\alpha}\left(X_{d}\right)\right)+K
\end{array}
$$

where the latter inclusion turns into an equality if $K=\mathbb{R}_{+}^{d}$. If $K=A^{-1} \mathbb{R}_{+}^{d}$ for a matrix $A$, then

$$
s_{\alpha}(X)=A^{-1}\left(\mathrm{ES}_{\alpha}\left((A X)_{1}\right), \mathrm{ES}_{\alpha}\left((A X)_{2}\right), \ldots, \mathrm{ES}_{\alpha}\left((A X)_{d}\right)\right)+K
$$

where $(A X)_{i}$ stands for the $i$ th coordinate of $A X$. In particular, (7.1) implies that the marginalized expected shortfall (as in Example 2.6) of $A X$ is coordinatewise smaller than $A$ applied to the marginalized expected shortfall of $X$. 
Risk measures generated by expected convex hull trimming The expected convex hull trimming induces coherent risk measures. Then $s_{1 / n}\left(X_{1}\right)=\left[\operatorname{EM}_{1 / n}\left(X_{1}\right),+\infty\right)$ and

$$
\begin{aligned}
s_{1 / n}\left(X_{1}, X_{1}, \ldots, X_{1}\right) & =\left(\mathrm{EM}_{1 / n}\left(X_{1}\right), \mathrm{EM}_{1 / n}\left(X_{1}\right), \ldots, \mathrm{EM}_{1 / n}\left(X_{1}\right)\right)+K, \\
s_{1 / n}(X) & \supseteq\left(\mathrm{EM}_{1 / n}\left(X_{1}\right), \mathrm{EM}_{1 / n}\left(X_{2}\right), \ldots, \mathrm{EM}_{1 / n}\left(X_{d}\right)\right)+K
\end{aligned}
$$

with equality if $K=\mathbb{R}_{+}^{d}$. If $K=A^{-1} \mathbb{R}_{+}^{d}$ for a matrix $A$, then (7.1) also holds for the expected minimum instead of the expected shortfall.

Note that in all three examples described above we have

$$
s_{\alpha}^{\mathrm{r}}(X) \supseteq\left(\rho\left(X_{1}\right), \ldots, \rho\left(X_{d}\right)\right)+K^{\mathrm{r}}
$$

where $\rho$ stands for $\mathrm{V} @ \mathrm{R}_{\alpha}, \mathrm{ES}_{\alpha}$ or $\mathrm{EM}_{1 / n}$.

Integral trimmed risk measures The integral trimmed regions generate new multivariate risk measures. Consider the cone $\mathbb{G}_{K}$ from Example 2.4 and $f(x)=-x+K$. Let $\mathcal{F}=\left\{\mathfrak{f}(t x+z): t>0, z \in \mathbb{R}^{d}\right\}$, where $\mathfrak{f}$ is continuous and $\leq_{K}$-decreasing for a proper Riesz cone $K$. Since $\mathrm{D}^{\alpha}(X)$ is the union of

$$
\mathrm{D}^{1}(Y)=\bigcap_{t>0, z \in \mathbb{R}^{d}}\left[\frac{1}{t}\left(\mathfrak{f}^{-1}(\mathbf{E} \mathfrak{f}(t Y+z))-z\right) \oplus K\right]
$$

for $Y \in \mathcal{P}_{\alpha}(X)$, we obtain

$$
s_{\alpha}(X)=\bigcap_{Y \in \mathcal{P}_{\alpha}(X)} s_{1}(Y)
$$

so that $s_{\alpha}(X)$ appears from the worst conditioning construction applied to the risk measure $s_{1}$. Furthermore, (6.5) yields that $s_{1}(X)=x+K$, where

$$
\begin{aligned}
x=-\wedge_{K} \mathrm{D}^{1}(X) & =-\wedge_{K}\left(\bigcap_{t>0, z \in \mathbb{R}^{d}}\left[\frac{1}{t}\left(\mathfrak{f}^{-1}(\mathbf{E} \mathfrak{f}(t Y+z))-z\right) \oplus K\right]\right) \\
& =-\bigvee_{t>0, z \in \mathbb{R}^{d}} \wedge_{K}\left[\frac{1}{t}\left(\mathfrak{f}^{-1}(\mathbf{E} \mathfrak{f}(t Y+z))-z\right)\right] \\
& =\bigwedge_{t>0, z \in \mathbb{R}^{d}}\left(-\wedge_{K}\left[\frac{1}{t}\left(\mathfrak{f}^{-1}(\mathbf{E} \mathfrak{f}(t Y+z))-z\right)\right]\right) .
\end{aligned}
$$

This risk measure satisfies R1-R3 and results from the homogenization construction (4.1) and (4.2) applied to the set-valued risk measure generated by the integral trimmed regions whose generating family is $\mathcal{F}=\{\mathfrak{f}\}$,

$$
\varrho(X)=-\wedge_{K} \mathfrak{f}^{-1}(\mathbf{E} \mathfrak{f}(X))+K
$$


Notice that this homogenization preserves R2, but not necessarily R4. The idea of constructing scalar risk measures using real-valued functions of vector portfolios appears also in [4]. Alternatively, it is possible to take the infimum in over $t>0$ or over $z \in \mathbb{R}^{d}$ only, which results in a risk measure that satisfies $\mathrm{R} 3$ or $\mathrm{R} 1$, respectively.

Example 7.1 The function $\mathfrak{f}(t)=\mathrm{e}^{-t / \gamma}$ yields the risk measure $\varrho(X)=$ $\gamma \log \left(\mathbf{E} e^{-X / \gamma}\right)$ by (7.3) in $\mathbb{G}=\mathbb{R}$ with the reversed order and $f(x)=-x$. The properties R1 and R2 evidently hold, while (2.2) follows from the Hölder inequality, i.e., $\varrho$ is a convex risk measure which does not satisfy R3. Since R1 already holds, there is no need to take the infimum over $z \in \mathbb{R}^{d}$ in (7.2). The corresponding convex risk measure is called the entropic risk measure with $\gamma$ being the risk tolerance coefficient.

If we attempt to produce a homogeneous (and thereupon coherent) risk measure from $\varrho$, we need to apply (4.1), which, in view of the reversed order on the real line, turns into

$$
\varrho_{\mathrm{h}}(X)=\sup _{t>0} t^{-1} \varrho(t X)=\sup _{t>0} t^{-1} \log \left(\mathbf{E} e^{-t X}\right)=\sup _{t>0} \log \left(\left(\mathbf{E} Y^{t}\right)^{1 / t}\right)
$$

for $Y=\mathrm{e}^{-X}$. Since $\left(\mathbf{E} Y^{t}\right)^{1 / t}$ is an increasing function of $t>0$, we have

$$
\varrho_{\mathrm{h}}(X)=\lim _{t \rightarrow \infty} t^{-1} \log \left(\mathbf{E} \mathrm{e}^{-t X}\right) .
$$

It is easy to see that the limit equals ( $-\operatorname{ess} \inf X$ ), so a coherent variant of $\varrho$ is not particularly interesting.

\section{Depth-trimmed regions generated by risk measures}

Consider a family of homogeneous risk measures $\varrho_{\alpha}$ for $\alpha \in(0,1]$ such that

$$
\varrho_{\alpha} \succeq \varrho_{\beta}, \quad \alpha \geq \beta,
$$

which are associated with a function $f$ according to R1. For instance, such a family of risk measures can be produced using the worst conditioning construction from Sect. 4.4 .

Definition 8.1 The depth-trimmed regions generated by the family of risk measures are defined as

$$
\mathrm{D}^{\alpha}(X)=\left\{y \in \mathbb{R}^{d}: \varrho_{\alpha}(X-y) \preceq \mathbf{e}\right\} .
$$

By R1, the depth-trimmed regions generated by a family of risk measures are alternatively given by

$$
\mathrm{D}^{\alpha}(X)=\left\{y \in \mathbb{R}^{d}: \varrho_{\alpha}(X) \preceq f(y)\right\} .
$$

Theorem 8.2 The depth-trimmed regions generated by a family of risk measures 
(i) satisfy properties $D 1, D 2, D 3$, and D5;

(ii) are convex if the risk measure is convex;

(iii) are closed if $f$ is upper semicontinuous, i.e., $\left\{x \in \mathbb{R}^{d}: a \preceq f(x)\right\}$ is closed in $\mathbb{R}^{d}$ for every $a \in \mathbb{G}$.

Proof (i) Properties D1 and D2 trivially hold by R1 and R3, respectively. The nesting property D3 of depth-trimmed regions is a consequence of (8.1). We show that D5 follows from R2. If $Y \leq_{K} X$ a.s., then R2 yields that $\varrho_{\alpha}(Y) \preceq \varrho_{\alpha}(X)$. Then

$$
\left\{y \in \mathbb{R}^{d}: \varrho_{\alpha}(Y) \preceq f(y)\right\} \supseteq\left\{y \in \mathbb{R}^{d}: \varrho_{\alpha}(X) \preceq f(y)\right\},
$$

and by (8.2) we have $\mathrm{D}^{\alpha}(Y) \supseteq \mathrm{D}^{\alpha}(X)$ and finally $\mathrm{D}^{\alpha}(X) \subseteq \mathrm{D}^{\alpha}(Y) \oplus K$, since $0 \in K$.

(ii) Given $y, z \in \mathrm{D}^{\alpha}(X)$ and $t \in[0,1]$,

$$
\varrho_{\alpha}(X) \preceq t \odot f(y) \oplus(1-t) \odot f(z)=f(t y+(1-t) z)
$$

and finally $t y+(1-t) z \in \mathrm{D}^{\alpha}(X)$

(iii) If $f$ is upper semicontinuous, the set $\left\{y \in \mathbb{R}^{d}: \varrho_{\alpha}(X) \preceq f(y)\right\}$ is closed.

Under mild conditions, it is possible to recover a risk measure from the depthtrimmed regions generated by it. If $\mathbb{F}$ is sup-generating and inf-generating, the original risk measure is the risk measure induced by the family of depth-trimmed regions that it generated. Theorem 6.5 and (8.2) yield that

$$
s_{\alpha}(X)=\bigwedge \mathbb{D}^{\alpha}(X)=\bigwedge\left\{f(y): \varrho_{\alpha}(X) \preceq f(y)\right\}=\varrho_{\alpha}(X) .
$$

Notice that if the construction based on rejection sets is used, see (3.3), the first equality in the above equation holds when $\mathbb{F}$ is inf-generating, so the sup-generating assumption on $\mathbb{F}$ can be dropped, and we still have

$$
s_{\alpha}^{\mathrm{r}}(X)=\varrho_{\alpha}(X) .
$$

Example 8.3 (Expected convex hull trimming revisited) The expected minimum can be formulated as a spectral risk measure, see [1], via

$$
\operatorname{EM}_{1 / n}(X)=-\int_{0}^{1} n(1-t)^{n-1} F_{X}^{-1}(t) \mathrm{d} t, \quad n \geq 1 .
$$

For any $\alpha \in(0,1]$, define $\operatorname{EM}_{\alpha}(X)$ substituting $n$ by $\alpha^{-1}$ in (8.3). The risk measures $\mathrm{EM}_{\alpha}$ generate a family of depth-trimmed regions for $X \in L_{1}^{\infty}$ with a continuous parameter $\alpha \in(0,1]$. Applying Definition 8.1, we obtain $\mathrm{D}^{\alpha}(X)=\left[-\operatorname{EM}_{\alpha}(X),+\infty\right)$.

In contrast to risk measures, depth-trimmed regions treat all directions in the same way, so that the regions $\mathrm{D}^{\alpha}$ must be slightly modified to ensure that they yield the expected convex hull trimmed regions for $\alpha=1 / n$. Define

$$
\begin{aligned}
\operatorname{CD}^{\alpha}(X) & =\mathrm{D}^{\alpha}(X) \cap\left(-\mathrm{D}^{\alpha}(-X)\right) \\
& =\left[-\operatorname{EM}_{\alpha}(X), \operatorname{EM}_{\alpha}(-X)\right] \\
& =\left[\alpha^{-1} \int_{0}^{1}(1-t)^{\alpha^{-1}-1} F_{X}^{-1}(t) \mathrm{d} t, \alpha^{-1} \int_{0}^{1} t^{\alpha^{-1}-1} F_{X}^{-1}(t) \mathrm{d} t\right] .
\end{aligned}
$$


In this formulation, we can assume that the parameter $\alpha$ takes any value in $(0,1]$ and thus we obtain an extension of the univariate expected convex hull trimmed regions.

\section{Duality results}

The dual space to $L_{d}^{\infty}$ is the family of finitely-additive bounded vector measures $\mu=\left(\mu_{1}, \ldots, \mu_{d}\right)$ on the underlying probability space $(\Omega, \mathfrak{F})$ which act on $X \in L_{d}^{\infty}$ as $\mathbf{E}_{\mu}(X)=\sum_{i=1}^{d} \int X_{i} \mathrm{~d} \mu_{i}$. The polar set to the cone of acceptable portfolios to a coherent risk measure $\varrho$ can be written as

$$
\mathcal{A}^{*}=\bigcap_{X \in \mathcal{A}}\left\{\mu: \mathbf{E}_{\mu}(X) \geq 0\right\} .
$$

As in [16], we can apply the bipolar theorem to show that

$$
\mathcal{A}=\bigcap_{\mu \in \mathcal{A}^{*}}\left\{X: \mathbf{E}_{\mu}(X) \geq 0\right\} .
$$

For each $u \in K$ and measurable $\Omega^{\prime} \subset \Omega$, the random element $u \mathbf{1}_{\Omega^{\prime}}$ belongs to $\mathcal{A}$. Therefore, every $\mu \in \mathcal{A}^{*}$ satisfies $\sum \mu_{i}\left(\Omega^{\prime}\right) u_{i} \geq 0$ for every $u \in K$. Thus, the values of any $\mu \in \mathcal{A}^{*}$ belong to the positive dual cone to $K$.

Assume that $\mathbb{F}$ is sup-generating. Proposition 3.1 implies that

$$
\varrho(X)=\bigvee\{f(y): X-y \in \mathcal{A}\} .
$$

From (9.1) it follows that

$$
\begin{aligned}
\varrho(X) & =\bigvee\left\{f(y): y \in \mathbb{R}^{d}, \mathbf{E}_{\mu}(X) \geq\langle\mu, y\rangle \text { for all } \mu \in \mathcal{A}^{*}\right\} \\
& =\bigvee f\left(\bigcap_{\mu \in \mathcal{A}^{*}}\left\{y \in \mathbb{R}^{d}:\langle\mu, y\rangle \leq \mathbf{E}_{\mu}(X)\right\}\right)
\end{aligned}
$$

where $\langle\mu, x\rangle=\sum_{i=1}^{d} \mu_{i}(\Omega) x_{i}$.

For instance, a set-valued risk measure with values in the cone of convex closed sets in $\mathbb{R}^{d}$ with the inclusion order (so that $f(-x)=x+K$ ) can be represented as

$$
\varrho(X)=\bigcap_{\mu \in \mathcal{A}^{*}}\left\{x \in \mathbb{R}^{d}:\langle\mu, x\rangle \geq \mathbf{E}_{\mu}(-X)\right\}
$$

where $\mathcal{A}^{*}$ is a set of finitely-additive bounded vector measures with values in $K$. Note that there is no need to add $K$ to the right-hand side, since for $z \in K$ it holds $\langle\mu, x+z\rangle \geq\langle\mu, x\rangle$ in case $\mu$ takes values from the positive dual to $K$. By applying to this set-valued risk measure $\varrho$ the worst conditioning construction, we obtain

$$
\begin{aligned}
\varrho_{\alpha}(X) & =\bigwedge_{Y \in \mathcal{P}_{\alpha}(X)} \bigcap_{\mu \in \mathcal{A}^{*}}\left\{x \in \mathbb{R}^{d}:\langle\mu, x\rangle \geq \mathbf{E}_{\mu}(-Y)\right\} \\
& =\bigcap_{\mu \in \mathcal{A}^{*}}\left\{x \in \mathbb{R}^{d}:\langle\mu, x\rangle \geq\left(-\mathbf{E}_{\mu}\right)_{\alpha}(X)\right\}
\end{aligned}
$$


where

$$
\left(-\mathbf{E}_{\mu}\right)_{\alpha}(X)=\left(-\mathbf{E}_{\mu_{1}}\right)_{\alpha} X_{1}+\cdots+\left(-\mathbf{E}_{\mu_{d}}\right)_{\alpha} X_{d} .
$$

Thus $\varrho_{\alpha}$ also admits a dual representation, where instead of the expectation $\mathbf{E}_{\mu}(-X)$ we take the expected shortfall of $X$ with respect to the measure $\mu$. Definition 8.1 then yields a dual representation for the family of depth-trimmed regions.

If the risk measure satisfies the Fatou property, then all measures from $\mathcal{A}^{*}$ can be chosen to be $\sigma$-additive. Recall that the Fatou property means that the risk measure is lower semicontinuous in probability, i.e., the lower limit (which for set-valued risk measures is understood in the Painlevé-Kuratowski sense [21, Definition B.5]) of $\varrho\left(X_{k}\right)$ is not greater than $\varrho(X)$ if $X_{k}$ converges in probability to $X$.

Example 9.1 (Vector-valued coherent risk measures) Let $\varrho$ be a coherent risk measure with values in $\mathbb{G}=\mathbb{R}^{d}$ with the reversed $\leq_{K}$-order with $K=\mathbb{R}_{+}^{d}$ and $f(x)=$ $-x$. Then

$$
\varrho(X)=\bigvee \bigcap_{\mu \in \mathcal{A}^{*}} Y(\mu),
$$

where all $\mu \in \mathcal{A}^{*}$ have nonnegative coordinates and

$$
Y(\mu)=\left\{-y:\langle\mu, y\rangle \leq \mathbf{E}_{\mu}(X), y \in \mathbb{R}^{d}\right\}=\left\{y:\langle\mu, y\rangle \geq-\mathbf{E}_{\mu}(X), y \in \mathbb{R}^{d}\right\} .
$$

For instance, the first coordinate of $\varrho(X)$ is obtained as the infimum of the projection of $\bigcap_{\mu \in \mathcal{A}^{*}} Y(\mu)$ onto the first coordinate. If $\mu_{1}(\Omega)>0$ and $\mu_{2}(\Omega)=\cdots$ $=\mu_{d}(\Omega)=0$, then $Y(\mu)=\left[y_{1}, \infty\right) \times \mathbb{R} \times \cdots \times \mathbb{R}$ for some $y_{1}$. If $x^{*}$ is the essential lower bound of $X$ with respect to the $\leq_{K}$-order, then $\left(y_{1},-x_{2}^{*}, \ldots,-x_{d}^{*}\right)$ belongs to $Y(\mu)$ for all $\mu \in \mathcal{A}^{*}$. Thus, the first coordinate of $\varrho(X)$ is given by the infimum $-\mu_{1}(\Omega)^{-1} \mathbf{E}_{\mu_{1}} X_{1}$ over all measures $\left(\mu_{1}, 0, \ldots, 0\right)$ that belong to $\mathcal{A}^{*}$. Therefore,

$$
\varrho(X)=\left(-\inf _{\mu_{1} \in \mathcal{A}_{1}^{*}} \mathbf{E}_{\mu_{1}}\left(X_{1}\right), \ldots,-\inf _{\mu_{d} \in \mathcal{A}_{d}^{*}} \mathbf{E}_{\mu_{d}}\left(X_{d}\right)\right)
$$

where $\mathcal{A}_{i}^{*}$ is the family of normalized measures $\mu_{i}$ such that $\left(0, \ldots, 0, \mu_{i}, 0, \ldots, 0\right) \in$ $\mathcal{A}^{*}, i=1, \ldots, d$. The individual infima in (9.2) are risk measures themselves. Thus, $\varrho(X)$ can be represented as the vector composed of coherent risk measures of the marginals of $X$.

A similar argument is applicable for the risk measure $A^{-1} \varrho(A X)$ if $K$ is a general Riesz cone given by $K=A^{-1} \mathbb{R}_{+}^{d}$, see Example 4.7. Then $A \varrho(X)$ can be represented as the vector composed of risk measures calculated for the coordinates of $A X$, cf. (7.1).

\section{Conclusions}

It is likely that other results from the morphological theory of lattices [13] have applications in the framework of risk measures. In particular, it would be interesting to find a financial interpretation for dilation mappings that commute with supremum, 
erosions that commute with infimum, and pairs of erosions and dilations that are called adjunctions.

It is possible to consider a variant of $\mathrm{R} 2$ where $Y \leq X$ is understood with respect to any other chosen order on $L_{d}^{\infty}$. The consistency issues for scalar risk measures for vector portfolios are investigated in [4] and [3] for the one-dimensional case.

Acknowledgements Ignacio Cascos acknowledges the hospitality of the Department of Mathematical Statistics and Actuarial Science of the University of Berne. The encouragement of the editors has led to a substantial improvement of the manuscript.

\section{References}

1. Acerbi, C.: Spectral measures of risk: A coherent representation of subjective risk aversion. J. Bank. Finance 26, 1505-1518 (2002)

2. Artzner, P., Delbaen, F., Eber, J.M., Heath, D.: Coherent measures of risk. Math. Finance 9, 203-228 (1999)

3. Bäuerle, N., Müller, A.: Stochastic orders and risk measures: consistency and bounds. Insur. Math. Econ. 38, 132-148 (2006)

4. Burgert, C., Rüschendorf, L.: Consistent risk measures for portfolio vectors. Insur. Math. Econ. 38, 289-297 (2006)

5. Cascos, I.: Depth functions based on a number of observations of a random vector. Working paper 07-29. Statistics and Econometrics Series, Universidad Carlos III de Madrid (2007). Available from http://econpapers.repec.org/paper/ctewsrepe/

6. Cascos, I., López-Díaz, M.: Integral trimmed regions. J. Multivar. Anal. 96, 404-424 (2005)

7. Cherny, A.S., Madan, D.B.: CAPM, rewards, and empirical asset pricing with coherent risk. Arxiv:math.PR/0605065 (2006)

8. Davydov, Y., Molchanov, I., Zuyev, S.: Strictly stable distributions on convex cones. ArXiv math.PR/0512196 (2005)

9. Delbaen, F.: Coherent risk measures on general probability spaces. In: Sandmann, K., Schönbucher, P.J. (eds.) Advances in Finance and Stochastics, pp. 1-37. Springer, Berlin (2002)

10. Embrechts, P., Puccetti, G.: Bounds for functions of multivariate risks. J. Multivar. Anal. 97, 526-547 (2006)

11. Föllmer, H., Schied, A.: Convex measures of risk and trading constraints. Finance Stochastics 6, 429447 (2002)

12. Hamel, A.H.: Translative sets and functions and their applications to risk measure theory and nonlinear separation. Available from http://www.gloriamundi.org (2006)

13. Heijmans, H.J.A.M.: Morphological Image Operators. Academic, Boston (1994)

14. Jarrow, R.: Put option premiums and coherent risk measures. Math. Finance 12, 135-142 (2002)

15. Jaschke, S., Küchler, U.: Coherent risk measures and good-deal bounds. Finance Stochastics 5, 181200 (2001)

16. Jouini, E., Meddeb, M., Touzi, N.: Vector-valued coherent risk measures. Finance Stochastics 8, 531552 (2004)

17. Kabanov, Y.M.: Hedging and liquidation under transaction costs in currency markets. Finance Stochastics 3, 237-248 (1999)

18. Koshevoy, G.A., Mosler, K.: Zonoid trimming for multivariate distributions. Ann. Stat. 25, 1998-2017 (1997)

19. Luxemburg, W.A.J., Zaanen, A.C.: Riesz Spaces, vol. 1. North-Holland, Amsterdam (1971)

20. Massé, J.C., Theodorescu, R.: Halfplane trimming for bivariate distribution. J. Multivar. Anal. 48, 188-202 (1994)

21. Molchanov, I.: Theory of Random Sets. Springer, London (2005)

22. Mosler, K.: Multivariate Dispersion, Central Regions and Depth. The Lift Zonoid Approach. Lecture Notes in Statistics, vol. 165. Springer, Berlin (2002)

23. Rousseeuw, P.J., Ruts, I.: The depth function of a population distribution. Metrika 49, 213-244 (1999)

24. Zuo, Y., Serfling, R.: General notions of statistical depth function. Ann. Stat. 28, 461-482 (2000)

25. Zuo, Y., Serfling, R.: Structural properties and convergence results for contours of sample statistical depth functions. Ann. Stat. 28, 483-499 (2000) 